\title{
SOME RESULTS ON DISJOINT STEINER QUADRUPLE SYSTEMS
}

\author{
CHIN-MEI FU AND HUNG-LIN FU
}

\section{Introduction}

A Steiner quadruple system of order $v, S Q S(v)$, is a pair $(Q, q)$, where $Q$ is a $v$-set and $q$ is a collection of 4-element subsets of $Q$, called blocks such that every 3-element subset is contained in exactly one block of $q$. H. Hanani [11] proved that an $S Q S(v)$ exists if and only if $v \equiv 2$ or $4(\bmod 6)$. Two Steiner quadruple systems $\left(Q, q_{1}\right)$ and $\left(Q, q_{2}\right)$ are said to intersect in $k$ blocks provided that $\left|q_{1} \cap q_{2}\right|=k$. The intersection problem of $S Q S(v)$ is to determine for which $k$ there exist two $S Q S(v)$ which intersect in $k$ blocks. Let $J[v]$ be the set of all nonnegative integers $k$ such that there exists a pair of $S Q S(v)$ which intersect in $k$ blocks. It was proved by M. Gionfriddo and C.C. Lindner that $J[v] \subseteq I[v]=\left\{0,1,2, \ldots, q_{v}-13, q_{v}-12\right\} \cup\left\{q_{v}-8, q_{v}\right\}$ where $q_{v}=v(v-1)(v-2) / 24$. At the same time they conjectured that $J[v]=I[v]$ for each $v \equiv 2$ or $4(\bmod 6)$. This conjecture is far from being solved. Since it is important to know first whether $0 \in J[v]$ or not, the existence of two disjoint $S Q S(v)$ becomes interesting.

In [13], C.C. Lindner and A. Rosa have shown that there exists a pair of disjoint $S Q S(v)$ whenever $v \equiv 4$ or $8(\bmod 12)$. For the case $v>14$ and $v \equiv 2$ or $10(\bmod 12)$, K.T. Phelps and A. Rosa [14] showed that there exist $n$ mutually disjoint $S Q S(2 n)$ for each $n=5^{a} \cdot 13^{b} \cdot 17^{c}$ for all $a, b, c \geq 0$ by using the construction of 2-chromatic $S Q S(2 n)$. But, in general, whether there exists a pair of disjoint $S Q S(v)$ when $v \equiv 2$ or $10(\bmod$ $12)$ is unknown. In this paper, we study the intersection problem [2-10] of $S Q S(3 v-2)$, $v \equiv 2$ or $4(\bmod 6)$, and we construct a pair of disjoint $S Q S(v)$ for each $v \equiv 46(\bmod$
$72)$.

\section{The main theorems}

A latin cube $\mathrm{C}$ of order $n$ is an $n$-tuple $\left(L_{1}, L_{2}, \ldots, L_{n}\right)$ of pairwise disjoint latin squares of order $n$; where two latin squares are disjoint if they have different elements at $(i, j)$ th entries for any $i$ and $j$. Two latin cubes $\left(L_{1}, L_{2}, \ldots, L_{n}\right)$ and $\left(M_{1}, M_{2}, \ldots, M_{n}\right)$
are disjoint if $L_{i}$ and $M_{i}$ are disjoint for $1 \leq i \leq n$.

A Steiner triple system of order $u(S T S(u))$ is a pair $(T, t)$ where $T$ is a $u$-set and $t$ is a collection of 3 -element subsets of $T$, called triples, such that every 2-element subset

Received June 26, 1989; revised February 26, 1990. 
of $T$ is contained in exactly one triple of $t$. It is well-known that an $S T S(u)$ exists if and only if $u \equiv 1$ or $3(\bmod 6)$. Also, for every $S T S(u)$, there is a collection of disjoint triples (partial parallel class) such that at least $\frac{u-1}{2}$ elements of $T$ are in these triples.

We now introduce the doubling construction of $S Q S$. Let $(X, A)$ and $(Y, B)$ be any two $S Q S(v)$ where $X \cap Y=\emptyset$. Let $F$ and $G, F=\left\{F_{1}, F_{2}, \ldots, F_{v-1}\right\}$ and $G=$ $\left\{G_{1}, G_{2}, \ldots, G_{v-1}\right\}$, be any two 1 -factorizations of $K_{v}$ on $X$ and $Y$, respectively, and let $\alpha$ be any permutation on the set $\{1,2, \ldots, v-1\}$. Define a collection of blocks $q$ on $Q=X \cup Y$ as follows:

(1) Any block belonging to $A$ or $B$ belongs to $q$, and

(2) If $x_{1}, x_{2} \in X$ and $y_{1}, y_{2} \in Y$ then $\left\{x_{1}, x_{2}, y_{1}, y_{2}\right\} \in q$ if and only if $\left\{x_{1}, x_{2}\right\} \in F_{i}$, $\left\{y_{1}, y_{2}\right\} \in G_{j}$ and $i \alpha=j$.

It is not difficult to check that $(Q, q)$ is an $S Q S(2 v)$.

Before we go any further, we need the following construction.

\section{Construction $\mathbb{F}$.}

Let $(P, p)$ be an $S Q S(v)$ such that $g \in P$. Set $Q=\{(a, i),(b, j),(c, k): i, j, k \in$ $P \backslash\{g\}\} \cup\{\infty\}$. Define a collection of blocks $q$ on $Q$ as follows:

(i) $\{\infty,(a, i),(b, i),(c, i)\} \in q$ for every $i \in P \backslash\{g\}$; and

(ii) $\{\infty,(x, i),(y, j),(z, k)\} \in q$ if $\{g, i, j, k\} \in p$, and $\{x, y, z\}=\{a, b, c\}$; and

(iii) $\{\infty,(x, i),(x, j),(x, k)\} \in q$ if $\{g, i, j, k\} \in p$ and $x \in\{a, b, c\}$; and

(iv) $\{(x, i),(x, j),(y, k),(z, k)\} \in q$ if $\{g, i, j, k\} \in p$ and $\{x, y, z\}=\{a, b, c\}$; and

(v) $\{(x, m),(x, n),(y, m),(y, n)\} \in q$ if $\{x, y\} \subseteq\{a, b, c\}$ and $\{m, n\} \subset P \backslash\{g\}$; and

(vi) $\{(x, h),(y, l),(z, m),(w, n)\} \in q$ if $x, y, z, w \in\{a, b, c\}$ and $\{h, l, m, n\}(h<l<m<$

$n$ ) not containing $g$ is a block in $p$ such that in the latin cube of order $3, C_{\{h, l, m, n\}}$, the $(x, y)$ enty of $L_{z}$ is $w$.

(We note here that we define a latin cube of order 3 corresponding to each block of $p$ which does not contain the element $g$.)

It is a routine matter to check that $(Q, q)$ is an $S Q S(3 v-2)$. Moreover, this $S Q S(3 v-$ 2) contains several subsystems of order 10 .

A partial quadruple system $(P Q S)$ is a pair $(S, s)$ where $S$ is finite set and $s$ is a collection of 4-element subsets of $S$ (called blocks) such that every 3-subset of $S$ is contained in at most one block of $s$. Two partial quadruple system $\left(S, s_{1}\right)$ and $\left(S, s_{2}\right)$ are said to be mutually balanced, if any given triple of distinct elements of $S$ is contained in a block of $s_{1}$ if and only if it is contained in a block of $s_{2}$. Two mutually balanced PQSs are disjoint if they have no block in common. Briefly, we denote two disjoint mutually balanced partial quadruple systems by DMB PQSs.

It is not difficult to see the following collection of 18 blocks $r_{\{i, j, k\}}$ (figure 2.1) and the collection of 18 blocks obtained in (iv) and ( $\mathrm{v}$ ) (Construction $\mathrm{F}$ ) with $\{i, j, k\}$ fixed and $\{m, n\} \subseteq\{i, j, k\}$ are DMB PQSs. 


$$
\begin{aligned}
& \{(a, i),(b, i),(b, j),(a, k)\},\{(a, i),(b, i),(a, j),(c, j)\}, \\
& \{(b, i),(c, i),(c, j),(b, k)\},\{(b, i),(c, i),(b, j),(a, j)\}, \\
& \{(c, i),(a, i),(a, j),(c, k)\},\{(c, i),(a, i),(c, j),(b, j)\}, \\
& \{(a, j),(b, j),(b, k),(a, i)\},\{(a, j),(b, j),(a, k),(c, k)\}, \\
& \{(b, j),(c, j),(c, k),(b, i)\},\{(b, j),(c, j),(b, k),(a, k)\}, \\
& \{(c, j),(a, j),(a, k),(c, i)\},\{(c, j),(a, j),(c, k),(b, k)\}, \\
& \{(a, k),(b, k),(b, i),(a, j)\},\{(a, k),(b, k),(a, i),(c, i)\}, \\
& \{(b, k),(c, k),(c, i),(b, j)\},\{(b, k),(c, k),(b, i),(a, i)\}, \\
& \{(c, k),(a, k),(a, i),(c, j)\},\{(c, k),(a, k),(c, i),(b, i)\},
\end{aligned}
$$

Figure 2.1.

Since we can construct two latin cubes of order 3 which are disjoint and there are $(v-1)(v-2) / 6$ triples $\{i, j, k\}$ such that $\{g, i, j, k\} \in p$, hence we have the following results.

Lemma 2.1. If $v \equiv 2$ or $4(\bmod 6)$, then $(3 v-3)(3 v-4) / 6+18 n \in J[3 v-2]$ for $n=0$ or $(v-1)(v-2) / 6$.

Proof. It is a direct result of Construction F.

As a matter of fact, if $(P, p)$ is an $S Q S(v)$, and $g \in P$, then the set $P \backslash\{g\}$ and the collection $t$ of all triples $\{i, j, k\}$ such that $\{g, i, j, k\} \in p$ forms an $\operatorname{STS}(v-1)$. (Derived triple system). Also, if $\left(P, p_{1}\right)$ and $\left(P, p_{2}\right)$ are disjoint $S Q S(v)$, then $\left(P \backslash\{g\}, t_{1}\right\}$ and $\left(P \backslash\{g\}, t_{2}\right)$ are disjoint $\operatorname{STS}(v-1)$.

Lemma 2.2. If there exist two disjoint $S Q S(v)$, then $v-1 \in J[3 v-2]$ where $v \equiv$ 2 or $4(\bmod 6)$.

Proof. We start with two SQS $(3 v-2),\left(Q, q_{1}\right)$ and $\left(Q, q_{2}\right)$, obtained from Construction $\mathrm{F}$ with two disjoint $\mathrm{SQS}(v),\left(P, p_{1}\right)$ and $\left(P, p_{2}\right)$. It is not difficult to see the possible blocks which $\xi_{2}$. and $q_{2}$ have in common are those blocks in (i) and (v) of Construction F. Now we replace , he collection of blocks from (iv) and (v) of $q_{1}$ by its DMB PQSs $r_{\{i, j, k\}}$ (Figure 2.1) for every triple $\{i, j, k\} \in t_{1}$, then we have the proof.

A parallel class of an $\operatorname{STS}(v)$ is the collection of disjoint triples which partition the $v$-set. A bit of reflection, $v \equiv 3(\bmod 6)$.

Lemma 2.3. If there exists a pair of disjoint $S Q S(v),\left(P, p_{1}\right)$ and $\left(P, p_{2}\right)$, such that the derived triple system $\left(P \backslash\{g\}, t_{2}\right)$ has a parallel class, then $0 \in J[3 v-2]$.

Proof. From Lemma 2.2, we have two SQS $(3 v-2),\left(Q, q_{1}\right)$ and $\left(Q, q_{2}\right)$ which have exactly $v-1$ blocks (i ) in common. Since $\left(P \backslash\{g\}, t_{2}\right)$ has a parallel class $\left\{\left\{i_{1}, j_{1}, k_{1}\right\}\right.$, 
$\left.\left\{i_{2}, j_{2}, k_{2}\right\}, \ldots\left\{i_{m}, j_{m}, k_{m}\right\}\right\}$ where $m=(v-1) / 3$, hence $\left(Q, q_{2}\right)$ has $m$ subsystems of order 10 which have one point in common. We can, of course, replace these SQS(10) with other SQS(10) independently [12]. For each $n=1,2, \ldots,(v-1) / 3$, we replace the subsystem, $\left(R_{n}, r_{n}\right)$ based on $\left\{\infty,\left(a, i_{n}\right),\left(b, i_{n}\right),\left(c, i_{n}\right),\left(a, j_{n}\right),\left(b, j_{n}\right),\left(c, j_{n}\right),\left(a, k_{n}\right),\left(b, k_{n}\right)\right.$, $\left.\left(c, k_{n}\right)\right\}$ by the SQS $(10),\left(R_{n},\left(r_{n}\right)_{\alpha}\right)$ where $\alpha$ is the permutation $\left(\left(a, i_{n}\right)\left(a, j_{n}\right)\left(a, k_{n}\right)\right)$ and $\left(r_{n}\right)_{\alpha}$ is the collection of blocks obtained by permuting the blocks of $r_{n}$ with $\alpha$. This gives the proof that $0 \in J[3 v-2]$. (We omit the detail of checking that each block in $\left(r_{n}\right)_{\alpha}$ is not in $q_{1}$.)

Recently, D. Woolbright [15] has shown that for each 1-factorization $\mathrm{F}$ of $K_{2 n}(n \geq$ 8), there exists a 1-factor with all its edges in distinct 1 -factors of $\mathrm{F}$. Hence we have the following lemma.

Lemma 2.4. If $v \equiv 2(\bmod 6)$, then there exists an $S Q S(2 v)(P, p)$ obtained from the doubling construction such that the derived $S T S(2 v-1),(P \backslash\{g\}, t)$, contains a parallel class.

Proof. Let $P=X \cup Y, X \cap Y=\emptyset,|X|=|Y|=v$, and $g \in X$. Since $v \equiv 2(\bmod$ $6)$, there exists an $\operatorname{SQS}(v),(X, A)[11]$. Then $\left(X \backslash\{g\}, A^{\prime}\right)$ is an $\operatorname{STS}(v-1)$ where $A^{\prime}=$ $\left\{\{(i, j, k\}:\{i, j, k, g\} \in A\}\right.$. It is well-known that at least $\frac{(v-1)-1}{2}$ elements of $X \backslash\{g\}$ is covered by a collection of disjoint triples of $A^{\prime}$, denote them by $x_{1}, x_{2}, \ldots, x_{(v-2) / 2}$. It is not difficult to see there are $\frac{v}{2}$ elements of $X \backslash\{g\}$ left. We can suitably choose $\alpha$ to match these $\frac{v}{2}$ elements to the $\frac{v}{2} 1$-factors which contain the edges of the 1 -factor produced by [15]. This will give us an $\operatorname{SQS}(2 v)(P, p)$ such that $(P \backslash\{g\}, t)$ contains a parallel class.

Lemma 2.5. If $v \equiv 16$ (mode 24), then there exists a pair of disjoint $S Q S(v),\left(P, p_{1}\right)$ and $\left(P, p_{2}\right)$, such that the derived $S T S(v-1),\left(P \backslash\{g\}, t_{2}\right)$, contains a parallel class. $(g \in$ P).

Proof. In [13]. it has been proved that $0 \in J[u]$ if $u \equiv 8(\bmod 12)$. By Lemma 2.4 , there exists an $\operatorname{SQS}(v),\left(P, p_{2}\right), v \equiv 16(\bmod 24)$, obtained from the doubling construction such that $\left(P \backslash\{g\}, t_{2}\right)$ contains a parallel class. Since $0 \in J[u]$ and there exists a pair of disjoint 1-factorizations of $K_{u}[1]$, by doubling construction, we can construct another $\operatorname{SQS}(v),\left(P, p_{1}\right)$, such that $\left(P, p_{1}\right)$ and $\left(P, p_{2}\right)$ have no blocks in common, this concludes the proof.

Theorem 2.6. There exists a pair of disjoint $S Q S(72 k+46)$ for every nonnegative integer $k$.

Proof. From Lemma 2.3, and Lemma 2.5.

\section{Remark}

A bunch of intersections on the set $J[72 k+46]$ and $J[3 v-2]$ can be obtained by unplugging and plugging DMB PQSs, since we cannot find the set $J[72 k+46]$ at this
time, we will not work on the details. 


\section{A.cknowledgement}

We would like to thank the referee's helpful comments and patience in correcting errors.

\section{References}

[1] H. L. Fu, "On the constructions and applications of two 1-factorizations with prescribed intersections", Tamkang J. of Math., Vol. 15, No. 1, 1984. [2] H. L. Fu, "Steiner quadruple systems of order $4 v$ with prescribed intersections", Ars Combinatoria,
21 (1986), pp.89-103.

[3] H. L. Fu, "A note on the block intersection problem of Stenier quadruple systems of order $u \cdot v$ ", Tamkang J. Math., Vol. 18, No. 2, 1987, pp. 59-63.

[4] H. L. Fu, "Intersection problem of Steniner systems S(3,4,2v)", Discrete Math., 67 (1987) 241-247.

[5] M. Gionfriddo and C.C. Lindner, "Construction of Steiner quadruple systems having a prescribed number of blocks in common", Discrete Math., 34 (1981), 34-42. [6] M. Gionfriddo, "On the set $J(v)$ for Steniner quadruple systems of order $v=2^{n}$ with $n \geq 4$ ",
Discrete Math., 44 (1983) $155-160$.

[7] M. Gionfriddo, "ON the block-intersection problem for SQS", Ars Combinatoria 15 (1983), 304-314. [8] M. Gionfriddo, "Intersections of Steiner systems $S(3,4, v)$ with $v=5 \cdot 2^{n}$ ", Journal of Geometry
24 (1985), 103-111. [9] M. Gionfriddo, M.C. Marino, "On Steniner systems $S(3,4,20)$ and $S(3,4,32)$ ", Utilitas Mathemat-
ices 25 (1984), 331-338. [10] Giovanni Lo Faro, "On the set $J(v)$ for Steiner quadruple systems of order $v=7 \cdot 2^{n}$ with $n \geq 2$ ",
Ars Combinatoria, Vol. $17(1984)$, pp. 39-47.

[11] H. Hanani, "On quadruple systems", Canad. J. Math. 12 (1960), 145-157. [12] E. S. Kramer amd D. M. Mesner, "Intersections among Steiner systems", J. Combinatorial Theory
16(A) (1974), 303-306. [13] C. C. Lindner and A. Rosa, "Finite embedding theorems for partial Steiner quadruple systems",
Bull. Soc. Math. Belg. 27 (1975) 315-323.

[14] K.T. Phelps and A. Rosa, "2-chromatic Steiner quadruple systems", Europ. J. Combinatorics (1980) 1, 253-258.

[15] D. Woolbright, "The rainbow theorem of 1-factorization", personal communication.

Chin-Mei Fu

Department of Mathematices, Tamkang University, Tamsui, Taiwan, ROC.

Hung-Lin Fu.

Department of Applied Mathematices, National Chiao Tung University, Hsin-Chu, Taiwan, ROC. 